\title{
A novel hybrid face recognition framework based on a low-resolution camera for biometric applications
}

\author{
Vijaya Kumar H. R., M. Mathivanan \\ Department of ECE, ACS College of Engineering, Visvesvaraya Technological University (VTU), Bengaluru, India
}

\begin{tabular}{l}
\hline \hline Article Info \\
\hline Article history: \\
Received May 8, 2021 \\
Revised Sep 16, 2021 \\
Accepted Sep 23, 2021 \\
\hline
\end{tabular}

\section{Keywords:}

DDWT

DOG

FFT

IFS

LG

\begin{abstract}
In research work, human face recognition is an essential biometric symbol persistently continued so far due to its different levels of applications in society. Since the appearance of the human faces can have many variations due to issues like the effect of illumination, expression and face pose. These differences are correlated with one another, which results in a helpless ability to recognize a particular person's face. The motivation behind our work in this paper is to give a new framework for face recognition based on frequency analysis that contributes to solving the distinguishing proof issues with enormous varieties of boundaries like the effect of illumination, expression, and face pose. Here three algorithms combined for provable results: i) Difference of Gaussian filtered discrete wavelet transform (DDWT) for feature extraction; ii) Log Gabor (LG) filter for feature extraction; and iv) Multiclass support vector machine classifier, where feature coefficients of DDWT and LG filter are fused for classification and parameters evaluation. The evaluation of our experiment is carried out on a large database consisting of 15 persons of each 200 -face image which are captured using a 5-megapixel low-resolution web camera and yielding satisfactory results on various parameters compared to existing methods.
\end{abstract}

This is an open access article under the CC BY-SA license.

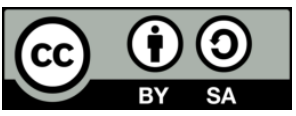

\section{Corresponding Author:}

M. Mathivanan

Department of ECE, ACS College of Engineering, Visvesvaraya Technological University (VTU)

Mysore Road, Kambipura, Bengaluru, Karnataka 560074, India

Email: mathivananacs@gmail.com

\section{INTRODUCTION}

Face recognition is a significant biometric attribute or symbol which helps to recognize a human face based on face detection, preprocessing, feature extraction, classification steps. The face detection method identifies the human face in a digital image. The preprocessing process suppresses noise or distortion content of the face image and trying to improve face image data values in detail and here both input and output are intensity valued face images. The feature extraction method is used to extract face features in terms of vector set coefficient points. The classification method is predictive modeling used to segregate feature vector setpoints into different categories. Even today, face recognition research is underway on a variety of new issues and new methods are being developed for different levels of applications. Saini and Kapoor [1] explained biometrics applications and challenges in forensic identification. The face recognition challenging pattern recognition problem in computing so that a lot of researchers were going for face recognition in the field of computer vision and presented many technologies, like biometrics in forensic identification. Gunasekaran et al. [2] proposed deep contourlet derivative weighted rank (DCD-WR) method using three significant attributes or symbols namely, face, iris, and fingerprint used for biometric applications. Singh and Prasad [3] reviewed the various techniques by considering challenging issues 
namely, pose variations, occlusion, expression, aging, environmental conditions. Sharif et al. [4] were used Gabor wavelets, neural network (NN), support vector machine (SVM), and Hidden markov model (HMM) methods for face recognition and here critical applications such as widespread computing, access control, and video surveillance are discussed in detail. Chihaoui et al. [5] reviewed different biometric attributes or symbols: face, fingerprint, hand, eye, voice, and signature along with local, global, and hybrid feature approaches. Kortli et al. [6] reviewed the various face recognition techniques in two-dimensional (2D) or three-dimensional (3D) format based on local, holistic, and hybrid features, and these techniques were compared by showing their recognition rate, level of complexity, robustness, and discrimination. Lumini et al. [7] proposed a new framework for the face recognition system in a hybrid manner using various preprocessing, feature extraction, dimension reduction, and classifier techniques. Efendi et al. [8] proposed the real-time attendance monitoring system using Viola-Jones and Eigenface approaches with various parameters. Castrillon et al. [9] proposed an object detection framework using the Viola-Jones algorithm. Irgens et al. [10] implemented the Viola-Jones algorithm in field programmable gate arrays (FPGA) platform for video surveillance and tracking applications. Ali et al. [11] proposed the modified local binary pattern histogram (MLBPH) method for face detection and recognition in a video stream and here Haar cascades were used for skin, eye, and nose detection of the human face.

\section{RELATED WORKS}

Gao et al. [12] proposed corner detection using Eigenvalues and LG filter on multiscaled gray images. Elsayed et al. [13] proposed an image denoising algorithm for clinical diagnostic application by developing a kernel-based singular value decomposition (K-SVD) using regularized orthogonal matching pursuit (ROMP), LG, Gabor, and discrete cosine transform (DCT). Ratul et al. [14] combine the LG filter and the SVD algorithm for the extraction of texture features and the Naïve Bayes method for classification in the NVIDIA GeForce GTX780 GPU platform. Allagwail et al. [15] used the symmetry approach with local binary pattern (LBP), gray level coherence matrix (GLCM), Gabor filter and Euclidian distance classifier for face recognition. Here the difference of Gaussian (DOG), discrete wavelet transform (DWT) and Gaussian Filter methods are used for preprocessing. Ayyavoo and Suseela [16] proposed a DWT enhanced contrast adaptive histogram equalization (DWT E-CLAHE) with Gabor feature and linear discriminant analysis (LDA) classifier for face recognition and experimented on CMU-PIE, extended Yale B face databases. Here the Gamma intensity correction (GIC), logarithmic transform (LT), contrast adaptive histogram equalization (CLAHE) methods are used for enhancing images. The DWT method was used to obtain four frequency different segment bands namely, approximate segment band (LL), horizontal segment band (HL), vertical segment band (LH), and diagonal segment band (HH). Sujatha et al. [17] proposed an image translation mathematical model with Gaussian filter, approximate segment band (DWT (LL)) and SVM classifier methods. The experiment was conducted on japanese female facial expression (JAFFE), our database of faces (ORL), Indian, L speack face databases. Roopashri and Kulkarni [18] proposed DWT (LL) and fast fourier transform (FFT) approach with Euclidean distance classifier. In this work, the noise removing, smoothing and resizing $(256 * 256)$ steps were used for preprocessing. Benzaoui et al. [19] proposed an automated human identification method by combining one-dimensional local binary pattern (1D-LBP), DWT and SVM classifier. Here the experiment was conducted on ORL and AR face databases. Al-Abaji and Salih [20] were proposed one-dimensional data reduction techniques namely, GLCM, PCA, DWT with Euclidian distance classifier for face recognition. In this work, the PCA features, HH segment band DWT features, and the GLCM of energy, contrast, correlation, and homogeneity features were considered for the experiment. Setumin et al. [21] proposed the DOG technique which is used for face sketches to match face photos. The fiducial points were used to solve the issues of exaggeration shape and the DOG preprocessing technique was used to eliminate noise in the image. Anila and Devarajan [22] proposed a new face recognition scheme using gamma correction, DOG, contrast normalization preprocessing methods. In this work, the experiment is evaluated on Yale-B, face recognition grand challenge (FRGC) version 2, and own databases. Davidson and Abramowitz [23], presented the effectiveness of the DOG filtering method for 12 specimen irregular microscopic images. González and Woods [24] explained in detail about digital image processing. Sun [25] addressed a high-dimensional space feature classification known as a multiclass support vector machine. The performance has been evaluated on glass, wine, iris, and vehicle datasets using various kernel functions of the multiclass support vector machine. Sun et al. [26] were used various classification methods for the analysis of radiomics glioma grading by considering ROC, area under curve (AUC), and accuracy parameters. Here classification methods namely, multinomial Naive Bayes, Bernoulli Naïve Bayes, k-nearest neighbors $(\mathrm{KNN})$, random forest, boosting decision tree, LDA, regression, RBF support vector machine, polynomial support vector machine, AdaBoost, Xgboost, Bagging, decision tree, and multilayered layer perception were utilized. The multiclass support vector machine is a significant binary classifier based on 
four types of operations namely, all classes at one, error-correcting output code, pairwise, one against all. Silva [27] explained in detail about multiclass support vector machine classifier. Mahmoodi et al. [28] proposed the implementation of linear and nonlinear support vector machine in the FPGA platform. Ghrabat et al. [29] proposed a novel hybrid classifier for image retrieval by combining support vector machine with convolution neural network on the LFW, Te European 1M, Corel 1K, and Flickr 27 database. From the literature survey, the face recognition model can be developed by considering the whole human face image in the frequency domain to solve low-resolution camera issues like the effect of illumination, expression, and face pose. The Gabor filter method is used to generate the face features of an image in a particular wavelength and direction by capturing a relevant frequency range. The Gabor filter will have both non-zero DC components and small DC components at certain bandwidth ranges and suffers from bandwidth problems at different orientations of face images.

The LG filter can be worked with subjective bandwidth and advanced for separating the bandwidth with insignificant local inclusion. The LG filter solves the bandwidth limitation and also represents the properties of face images in terms of local frequency. The LG filter can be formed by combining two components based on the frequency and direction of face images. From the survey, the DWT method in the frequency domain is always considered to obtain four-segment bands where the approximate segment band called DWT (LL) is always considered to obtain image feature coefficients because it will produce detailed information. But LL segment band was not enhanced properly when it is outputted from the DWT face image due to missing some of the low or high-frequency components. Hence it is necessary to apply a filtering technique to each face image before applying DWT that helps to enhance the edges of face images properly without loss of low or high-frequency components of the image. The DOG filtering technique is a significant technique based on a Gaussian filter that may use to enhance the edges of face images. Another problem is that when considering large face images, it is necessary to normalize the gray values from 0 to 255 points for each face image in various levels to match the feature coefficients for all face images. Finally, a mathematical framework is required to obtain the DDWT and LG feature coefficient points that are integrated into the classification. From the survey, a multiclass support vector machine with RBF kernel function may be chosen for better classification performance using various parameters.

\section{METHODOLOGY}

The design of the proposed framework is shown in Figure 1. It includes five stages namely, face detection using Viola-Jones algorithm, pre-processing of detected face images, feature extraction using DDWT and LG filter, multiclass support vector machine with radial base function (RBF) kernel in non-linear fashion for classification, and parameters evaluation. The key point in the proposed framework is that both the training and testing of facial images follow the same procedure.

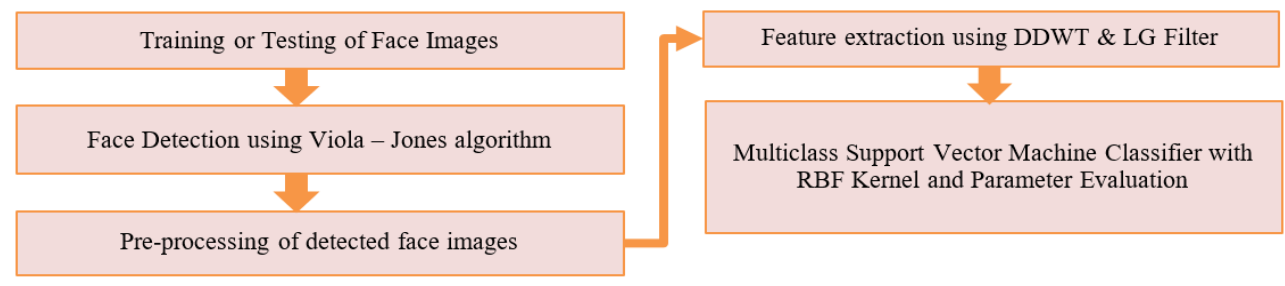

Figure 1. The design of the proposed framework

\subsection{Training and testing face images}

The database consists of 3000 face images of 15 individuals and each person consists of 200 face images. In this work, the training and testing of face images are divided into a ratio of 90:10, that is, 2700 face images are trained and 300 face images are examined.

\subsection{Face detection using Viola-Jones algorithm}

The Viola-Jones algorithm is a significant face detection method developed by Viola and Jones back in 2001 [8]-[11], [30]. The Viola-Jones algorithm follows three stages namely, haar classifier, integral image, and cascading classifier. The haar classifier is used to find features of each subsection in a given digital image. Next, an integral image is created by calculating the sum of all pixels inside four corners of the rectangle by above and left pixels. Finally, cascading classifiers are composed of several stages where all 
features are grouped with a certain amount of features that help to scan the image for identifying human face repeatedly with the new size. The Face detection process is shown in Figures 2 (a)-(c).

\subsection{Pre-processing of detected face images}

The Pre-processing method was used to improve facial image features by eliminating unnecessary distortion or noise. First, the detected color face image was converted into a greyscale face image. Then, contrast stretching of face image using low and high threshold values of the image with high and low pixel values is used to review for face image contrast. Finally, the normalized face image is resized to $128 \times 128$ square matrix images. The complete pre-processing process is shown in Figures 2(d)-(f).

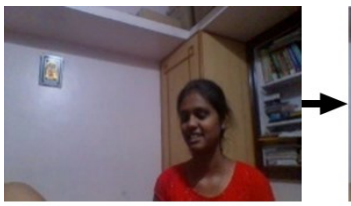

(a)

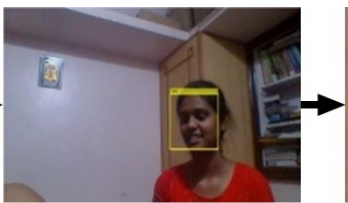

(b)

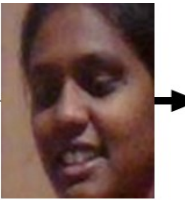

(c)

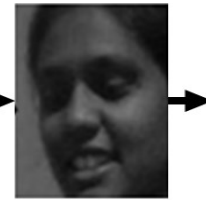

(d)

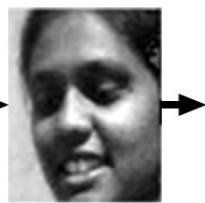

(e)

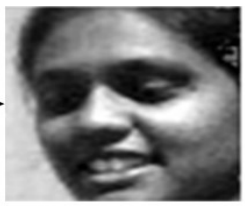

(f)

Figure 2. The Face detection process; (a) original image, (b) detected face, (c) cropped face image and the complete pre-processing process, (d) grey face image, (e) contrast stretching and (f) resize face image

\subsection{Difference of gaussian filtered discrete wavelet transform (DDWT) feature extraction}

The DDWT feature extraction consists of four stages, namely, difference of Gaussian (DOG), Single level discrete wavelet transform, normalization of face images, fast fourier transform (FFT), and image fourier shift (IFS). The DDWT Feature extraction is shown in Figure 3.

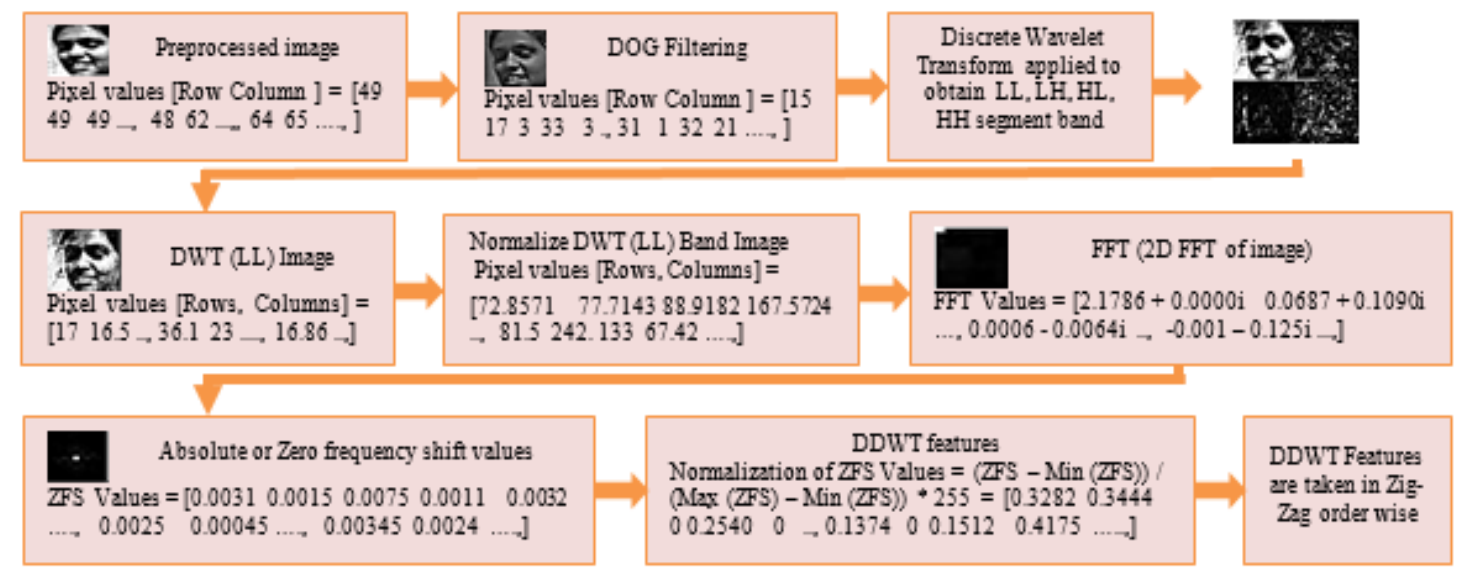

Figure 3. The DDWT feature extraction

\subsubsection{Difference of Gaussian (DOG)}

The DOG is the band-pass filter equivalent to the feature enhancement method that gives spatial structural information that lies between the set of frequency ranges [7], [15] [21]-[24]. Here one blurred version of the original image or preprocessed face image at a smaller kernel size is subtracted from another blurred version of the original image at a larger kernel size which helps to improve visibility of the edge and also presents other structured details present in a digital image. In 2-dimension, $\boldsymbol{\Gamma}$ which represents an image convoluted to the DOG and it is given in (1).

$$
\Gamma_{\sigma, \mathrm{K} \sigma}=\mathrm{I} *\left(\frac{1}{2 \pi \sigma^{2}} \mathrm{e}^{\frac{-\left(\mathrm{x}^{2}+\mathrm{y}^{2}\right)}{2 \sigma^{2}}}-\frac{1}{2 \pi \mathrm{K}^{2} \sigma^{2}} \mathrm{e}^{\frac{-\left(\mathrm{x}^{2}+\mathrm{y}^{2}\right)}{2 \mathrm{~K}^{2} \sigma^{2}}}\right)
$$

Where, image 1 is convolved with Gaussian of variance $\sigma 1$ and Gaussian narrow variance $\sigma 2$, with $\sigma 2>\sigma 1$, and $\mathrm{x}$ and $\mathrm{y}$ are coordinates. In this work $\sigma 1=0.5$ and $\sigma 2=1.5$ are assigned. 


\subsubsection{Single level discrete wavelet transform}

In this work, the single level DWT technique ( $1^{\text {st }}$ Level of DWT) is used to expand the DOG filtered face images into first level four frequency segment subband (LL, LH, HL, and HH) face images [15]-[19]. The 2D-DWT is given by (2) and (3).

$$
\begin{aligned}
& w_{\varphi}\left(j_{0}, m, n\right)=\frac{1}{\sqrt{M N}} \sum_{x=0}^{M-1} \sum_{y=0}^{N-1} f(x, y) \varphi_{j_{0}, m, n}(x, y) \\
& w_{\Psi}(j, m, n)=\frac{1}{\sqrt{M N}} \sum_{x=0}^{M-1} \sum_{y=0}^{N-1} f(x, y) \Psi_{j, m, n}^{i}(x, y)
\end{aligned}
$$

Where i subscript $=\{\mathrm{HL}, \mathrm{LH}, \mathrm{HH}\}, \mathrm{J} 0$ is arbitrary scaling factor, $w_{\varphi}\left(j_{0}, m, n\right)$ coefficient $(\mathrm{LL})$ of $\mathrm{f}(\mathrm{x}, \mathrm{y})$ at scale $\mathrm{J} 0, w_{\Psi}(j, m, n)$ coefficients add $(\mathrm{HL}),(\mathrm{LH})$, and $(\mathrm{HH})$ details for $\mathrm{J}>\mathrm{J} 0, \varphi_{j_{0}, m, n}(x, y)$ and $\Psi_{j, m, n}^{i}(x, y)$ are is scaled the translated functions. Due to contrast stretching high-frequency components contains more feature points with noisy sets. The approximate or detailed segment band-LL band or DWT (LL) which contains most visual parts of the face image and most signal energy, and hence here, only the DWT (LL) is considered by suppressing other frequency segment subbands, namely HH, HL, and LH.

\subsubsection{Normalization of face images}

All face images whose pixel values are not at the same value or uniformed condition due to their illumination effect, face pose, and expressions. These issues have a profound effect on the DWT (LL) face image, so here each DWT (LL) face image is normalized to gray values (0 to 255) and it is given by (4).

$$
P=255\left[\frac{\text { Rinput }- \text { Rmin }}{\text { Rmax }- \text { Rmin }}\right]
$$

Where $R_{\text {input }}$ is the intensity value of DWT (LL) face image, $R_{\min }$ is the minimum intensity value of DWT (LL) face image, $R_{\max }$ is the maximum intensity value of DWT (LL) face image.

\subsubsection{Fast fourier transform (FFT)}

The FFT was applied to get the ac energy coefficients from the normalized LL band image and was helped to obtain both real and imaginary values of the normalized DWT (LL) face image. The FFT converts the spatial domain into the frequency domain, and here magnitude is stored in the frequency domain [18]. In 2-dimension FFT is given by (5) and (6).

$$
\begin{aligned}
& \operatorname{ReX}[r, c]=\operatorname{Re} X[P-r, P-c] \\
& \operatorname{Im} X[r, c]=-\operatorname{Im} X[P-r, P-c]
\end{aligned}
$$

Where $\mathrm{P}$ is the test or sample number and $\mathrm{X}[\mathrm{r}, \mathrm{P}]$ is the deciphered as $\mathrm{X}[0,0], \mathrm{X}[\mathrm{r}, 0], \mathrm{X}[\mathrm{P}, \mathrm{c}], \mathrm{X}[0, \mathrm{C}], \mathrm{X}[\mathrm{P}$, $\mathrm{P}]$ and rage are situated at $(0,0),(0, \mathrm{P} / 1),(\mathrm{P} / 2,0)$ and $(\mathrm{P} / 2, \mathrm{P} / 2)$.

\subsubsection{Image fourier shift (IFS)}

The IFS is used to obtain zero frequency shift (ZFS) or Absolute values features by an outright estimation of the previous step (FFT real and imaginary values). Because of sine and cosine, work coefficients are unseemly henceforth it is important to standardize or normalize as in section 3.4.3, and that locates the most extreme and least qualities to include coefficients esteems. From these coefficient esteems highlights were chosen in the zig-zag order wisely as a DDWT features coefficients.

\subsection{Log Gabor (LG) filter feature extraction}

The LG filter is a significant logarithmic scale Gabor feature extraction method that is used by considering all frequency components in both real and imaginary parts [12]-[14]. The LG Filters has no DC components built with subjective bandwidth that can be streamlined to create a filter with a significant spatial degree. The LG filter feature extraction is shown in Figure 4.

The LG transfer function is represented,

$$
G(f)=\exp \left(\frac{-\left(\log \left(f / f_{o}\right)\right)^{2}}{2\left(\log \left(\sigma / f_{o}\right)\right)^{2}}\right)
$$


where $f_{0}$ is the center frequency of the filter, and $\sigma$ is the effects bandwidth, the ratio $\sigma /$ fo is filtered bandwidth approximately one octave if it is 0.74 , and 0.55 results in two octaves and 0.41 in three octaves such that it held constant for varying $\mathrm{f}_{\mathrm{o}}$.

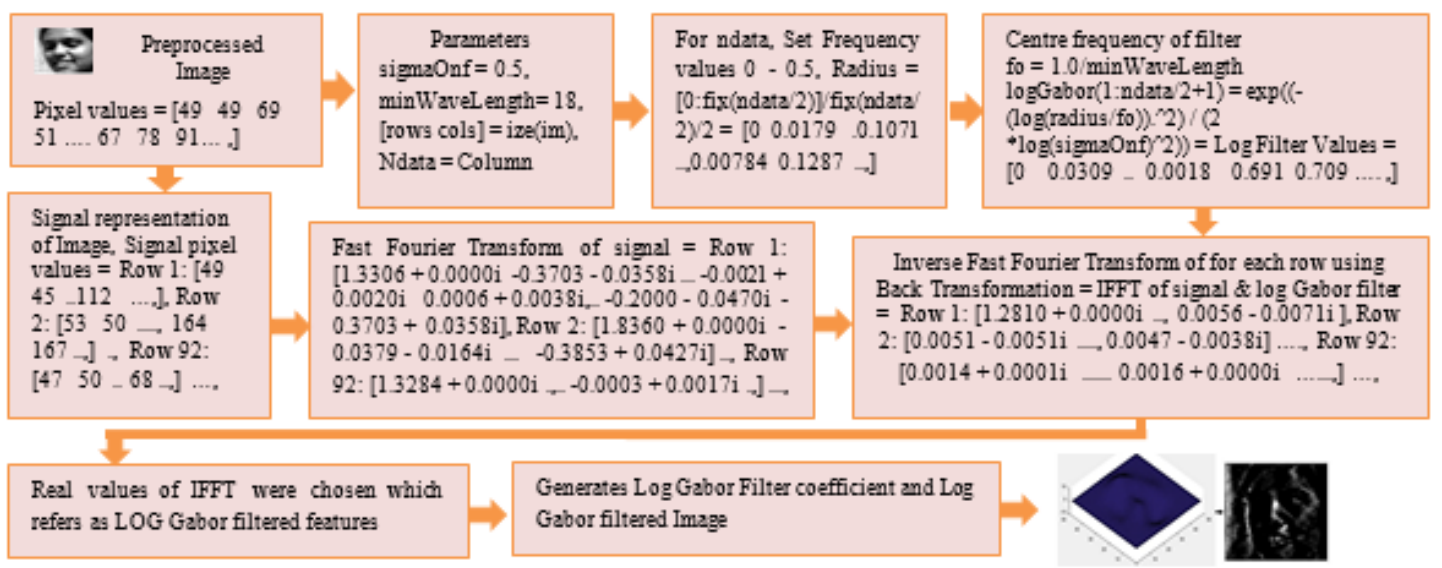

Figure 4. The LG feature extraction

\subsection{Multiclass support vector machine classifier}

The DDWT and LG feature coefficients are fused to a multiclass support vector machine classifier in a nonlinear fashion with an RBF kernel. The transformation function $\Phi: X \rightarrow F$ represents the point from the input space and maps all data points to a point in a new $\mathrm{F}$ space. The $\mathrm{X}_{\mathrm{i}}$ of $\mathrm{X}$ is represented as $\Phi(\mathrm{Xi})$ and dot product $\mathrm{x}^{\mathrm{T}} \mathrm{y}$ is represented as $(\Phi(\mathrm{X}), \Phi(\mathrm{y}))$ natural inner product function in new space $\mathrm{F}[25]-[28]$. The transformation function for nonlinear support vector machine with $\mathrm{RBF}$ is $\mathrm{k}: \mathrm{X}^{*} \mathrm{X} \rightarrow \mathrm{R}$. and the nonlinear support vector machine with RBF formula is as in (8) to (12). The optimal dividing hyperplane is given by,

$$
W=\sum_{i \in S V} h_{i} y_{i} k\left(x_{i}\right)
$$

Hyperplane function with transform function,

$$
\left(\mathrm{w}^{*}, \phi(\mathrm{x})\right)=\sum_{\mathrm{i} \in S V} \mathrm{~h}_{\mathrm{i}} \mathrm{y}_{\mathrm{i}} \mathrm{k}\left(\mathrm{x}_{\mathrm{i}}, \mathrm{x}\right)
$$

The offset of the hyperplane

$$
\begin{aligned}
& \mathrm{b}=\frac{1}{|\mathrm{SV}|} \sum_{\mathrm{i} \in S V}\left(\mathrm{y}_{\mathrm{i}}-\sum_{\mathrm{j}=1}^{\mathrm{n}}\left(\mathrm{h}_{\mathrm{i}} \mathrm{y}_{\mathrm{i}} \mathrm{k}\left(\mathrm{x}_{\mathrm{i}}, \mathrm{x}\right)\right)\right. \\
& \text { Classification rules }=\sum_{\mathrm{k}}^{\mathrm{Ns}}\left(\mathrm{h}_{\mathrm{i}} \mathrm{y}_{\mathrm{i}} \mathrm{e}^{\frac{\left\|\left(\mathrm{x}_{\mathrm{i}}-\mathrm{x}\right)\right\|^{2}}{\sigma^{2}}}+\mathrm{b}\right.
\end{aligned}
$$

where $\mathrm{X}$ is a list of training data vector points, $\mathrm{h}$ is the Lagrangian coefficients, and $\mathrm{SV}$ is a set of support vectors. The radial basis function (RBF) is defined,

$$
\mathrm{k}(\mathrm{x}, \mathrm{z})=\mathrm{e}^{\frac{\left\|\left(\mathrm{x}_{\mathrm{i}}-\mathrm{x}\right)\right\|^{2}}{2 \sigma^{2}}}, \text { where } \sigma 2>0
$$

where ||$\left(x_{i}-x\right) \|$ is Euclidean distance between any two vectors, and $\sigma$ is a parameter usually 1.

\section{RESULTS AND DISCUSSION}

All the face images were randomly captured using a 5-Megapixel low-resolution web camera at a distance of not more than 10 feet in both uniform and non-uniform nature. The captured face images will have various characteristics namely, the effect of Illumination conditions (high and low-intensity level), face pose (frontal, minor, and major pose), eyes conditions (close, open, slightly open, or close), and expression variations. The various samples of 200 face images of a single person with various characteristics are shown in Figure 5. 
The performance of the proposed face recognition system is executed in the MATLAB platform by considering the average of 10 iterations and was evaluated on 300 test face images by considering different measuring elements], such that true positives (TP): positive segments anticipated as positive; false positives (FP): negative segments anticipated as positive; false negatives (FN): positive segments anticipated as negative; true negatives $(\mathrm{TN})$ : negative segments anticipated as positive.

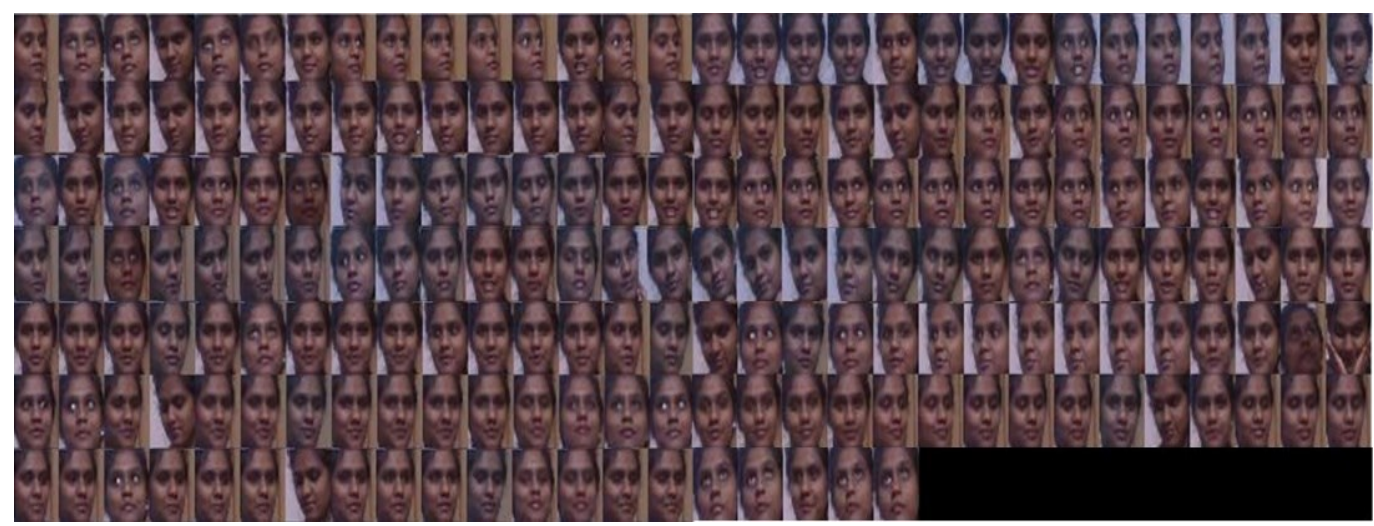

Figure 5. The various samples of 200 face images of a single person with various characteristics

The various parameters are evaluated by considering TP, TN, FP, and FN measuring elements are:

a) Accuracy (Acc): Accuracy is the ratio of amount effectively recognized as both positive and negative segments $(\mathrm{TP}+\mathrm{TN})$ to the total number of positive and negative elements $(\mathrm{TP}+\mathrm{TN}+\mathrm{FP}+\mathrm{FN})$. The Acc was used here to recognize the total number of exact individual face images from the total face images.

$$
A C C=\frac{T P+T N}{T P+T N+F P+F N}
$$

b) Recall (Rc): Recall or sensitivity or true positive rate of the classifier is the ratio of what amount was effectively recognized as positive (TP) segments to the certain amount (TP+FN) segments. Here $(\mathrm{TP}+\mathrm{FN})$ is the total amount of positive segments recognized exactly as positive (TP) and negative segments recognized as false $(\mathrm{FN})$.

$$
R c=\frac{T P}{T P+F N}
$$

c) Specificity (Sp): The specificity or false positive rate of the classifier is the ratio of what amount was effectively recognized as negative (TN) segments to what amount was negative (TN+FP) segments. Here $(\mathrm{TN}+\mathrm{FP})$ is the total amount of negative segments recognized as exactly as negative (TN) and positive segments recognized as false (FP).

$$
S p=\frac{T N}{T N+F P}
$$

d) Precision (Pr): The Precision of the classifier is referred to what amount were accurately delegated positive out of all positives. The Pr is the ratio of amount effectively recognized as positive segments (TP) to the total positive measuring segments $(\mathrm{TP}+\mathrm{FP})$ and which helps to predict exact positive element values as positive.

$$
\operatorname{Pr}=\frac{T P}{T P+F P}
$$

e) F1 and F2 scores: F1 and F2 scores are consonant normal of Sp and Pr. The F1 and F2 are calculated without considering TN values and shown the effectiveness of positive segments recognized as positive only out of all TP, FN, FN segments. In the F1 score, the Pr and Rc are balanced, and in the F2 score, Pr and Rc are not balanced such that here Rc is increased and Pr values are lowered that makes minimizes FN segments and also avoid FP segments.

$$
F 1=2 * \frac{(P r * R c)}{(\operatorname{Pr}+R c)}
$$




$$
F 2=\frac{(5 * \operatorname{Pr} * R c)}{(4 * \operatorname{Pr}+R c)}
$$

f) G1 and G2 scores: G1 score is the mathematical normal of recall and precision which and the G2 score is the mathematical normal of recall and specificity. The G1 and G2 scores are calculated by considering TN values also and give the effectiveness of exact positive value predictions concerning the certain amount of positive $(\mathrm{TP}+\mathrm{FN})$ segments for G1 score and negative ( $\mathrm{TN}+\mathrm{FP})$ segments for $\mathrm{G} 2$ score.

$$
\begin{aligned}
G 1 & =\sqrt{R c * P r} \\
G 2 & =\sqrt{R c * S p}
\end{aligned}
$$

g) Matthews correlation coefficient (MCC): The MCC is a more exact measurable rate that possibly gives a high score if the forecast has been delivered successfully on the whole four namely TP, TN, FP, and FN. The MCC rate of binary classification produces a high score in particular if the prediction had the option to effectively anticipate most of the positive data value cases and most of the negative data occasions. The scope of upsides of MCC lies between -1 to +1 and with a score of +1 is an ideal model and -1 is a helpless model. The MCC was used to identify exact positive segments by considering all false segments.

$$
M C C=\frac{(T P * T N)-(F P * F N)}{\sqrt{((T P+F P) *(T P+F N) *(T N+F P) *(T N+F N))}}
$$

h) Receiver operating characteristic (ROC) curve: The ROC curve is a 45-degree diagonal line graphical measuring plot used to identify the performance of the binary classifier. Here the X-axis is referred to as specificity, and the Y-axis is referred to as sensitivity. The ROC curve is depending on TP, TN, FN, and FP measuring element values, and the performance is said to be good whenever sensitivity values are at the improved level by diminishing specificity values.

The comparison results of the various parameters of the proposed method Vs existing methods are shown in Table 1 and the results of the ROC curve are shown in Figure 6. In DWT (LL) E-CLAHE+Gabor the ROC curve is very poor due to the large distribution of specificity values (45-degree diagonal lines). Next in the LG filter technique, the ROC curve shows moderate results with a certain degree of a small reduction in specificity values compared to the DWT (LL) E-CLAHE+Gabor method. Next is the Gaussian+DWT (LL) method which produces satisfactory results on all parameters and also the ROC curve was significantly improved compared to the DWT (LL) E-CLAHE+Gabor and LG filter methods.

Table 1. The comparison results of various parameters of the proposed method Vs existing methods

\begin{tabular}{lccccccccc}
\hline \multicolumn{1}{c}{ Method used } & \multicolumn{1}{c}{ Parameters } \\
& Acc & Rc & Sp & Pr & F1 & F2 & G1 & G2 & MCC \\
\hline DWT (LL) E-CLAHE+Gabor [16] & 0.911 & 0.869 & 0.914 & 0.381 & 0.523 & 0.68 & 0.57 & 0.89 & 0.53 \\
LG [12, 13, 14] & 0.914 & 0.933 & 0.912 & 0.442 & 0.595 & 0.758 & 0.639 & 0.923 & 0.605 \\
Gaussian+DWT (LL) [18] & 0.922 & 0.955 & 0.919 & 0.43 & 0.59 & 0.764 & 0.639 & 0.936 & 0.61 \\
DOG+DWT (LL)+LG & 0.82 & 0.943 & 0.807 & 0.261 & 0.405 & 0.613 & 0.493 & 0.872 & 0.435 \\
DWT (LL) \& LG & 0.927 & 0.96 & 0.92 & 0.501 & 0.66 & 0.81 & 0.69 & 0.94 & 0.66 \\
Gaussian+DWT (LL) \& LG & 0.93 & 0.956 & 0.928 & 0.482 & 0.638 & 0.795 & 0.677 & 0.942 & 0.659 \\
DDWT \& LG (Proposed) & 0.94 & 0.97 & 0.935 & 0.516 & 0.672 & 0.823 & 0.71 & 0.96 & 0.68 \\
\hline
\end{tabular}

In this work, firstly the DOG+DWT (LL) + LG is evaluated such that the DOG filter technique is applied to each face image and then for the DOG face image DWT is applied where LL band is only considered and then LG filter is applied on LL band to obtain feature coefficients, and these coefficients are fused to the multiclass support vector machine classifier. But here ROC curve produced the worst results due to the high impact of Specificity on Sensitivity. This might be happening due to the LG filter which is applied on DWT (LL) and cause loss of the detailed image feature information. Secondly, the DWT (LL) \& LG referring that the LL segment band of DWT and LG feature coefficients that are separately generated and that are fused to the multiclass support vector machine classifier, and here satisfactory results on all parameters were produced.

Thirdly, Gaussian DWT (LL) \& LG coefficients are separately generated and are fused to the multiclass support vector machine classifier. Here Gaussian was used for enhancing the face image and then for each Gaussian face image DWT was applied to get DWT (LL) face image. In this work, satisfactory results were obtained on all parameters, and also the ROC curve improved little compared to DWT (LL) and LG methods. Finally, the proposed DDWT and LG is referring that the DOG+DWT (LL) and LG features 
separately generated and fused to the multiclass support vector machine classifier. Here DOG was used for enhancing the preprocessed image and then DWT was applied to get the DDWT face image. The proposed method was proved significant results on all parameters compared to previous methods and also ROC curve is improved significantly by impacting sensitivity on specificity parameters compared to previous methods.

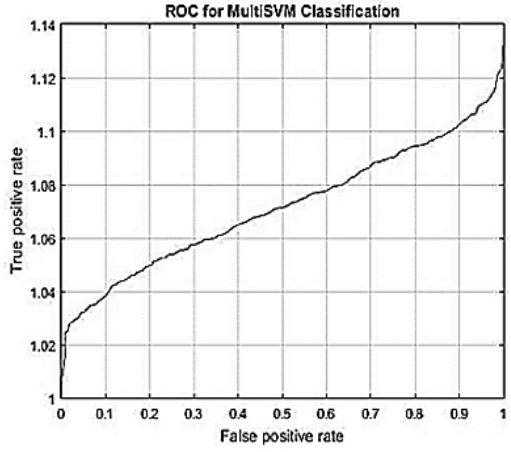

(a)

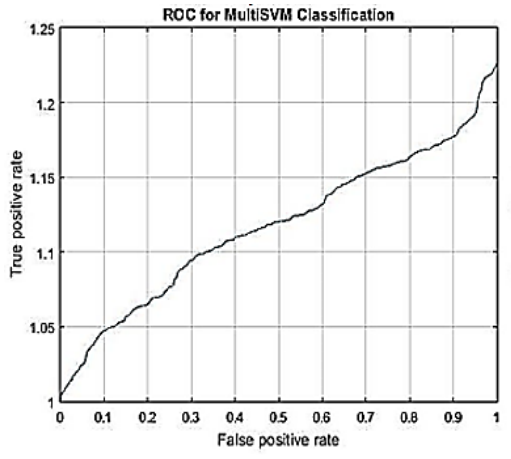

(d)

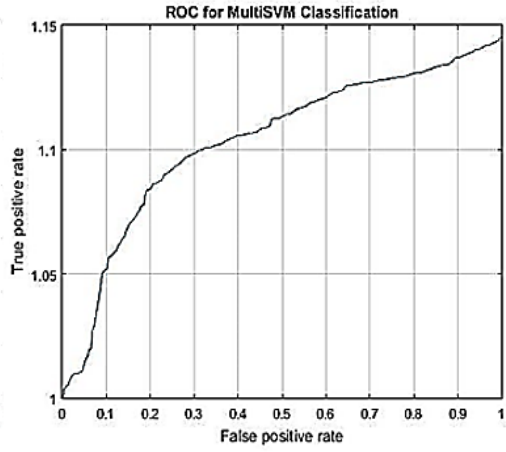

(b)

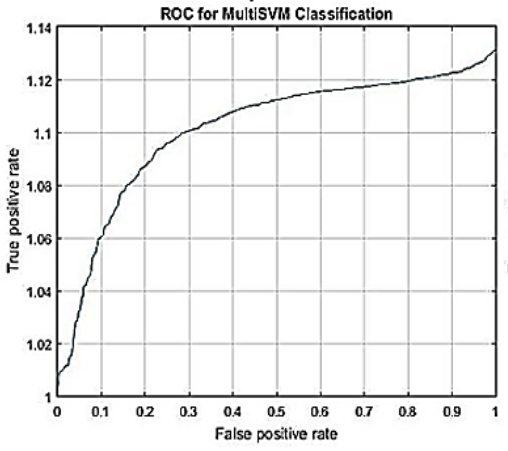

(e)

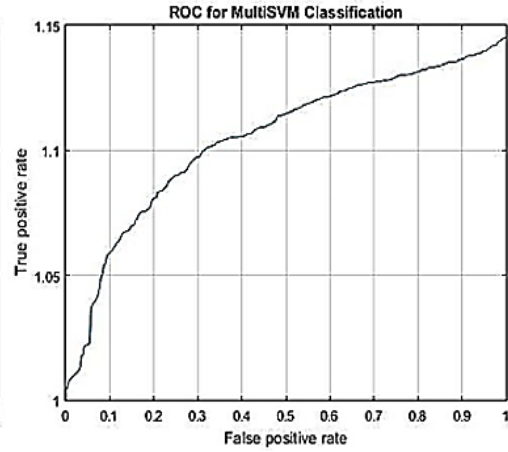

(c)

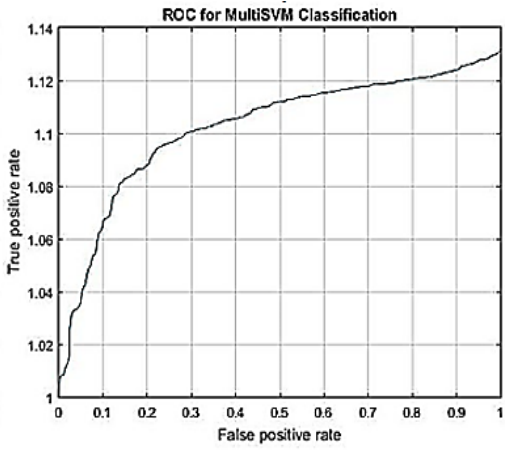

(f)

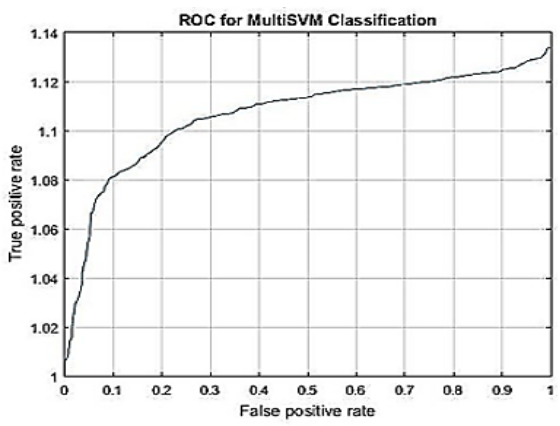

(g)

Figure 6. The results of ROC curve; (a) DWT (LL) E-CLAHE+Gabor, (b) LG, (c) Gaussian+DWT, (d) DOG+DWT (LL)+LG, (e) DWT (LL) and LG, (f) Gaussian+DWT (LL) and LG, and (g) DDWT and LG (proposed)

\section{CONCLUSION}

In this paper, a new face recognition model was proposed by combining DDWTand LG features with a nonlinear multiclass support vector machine with RBF Kernal which tried to solve the effects of lowresolution camera issues such as the effect of illumination, face pose, and expression. The evaluation of the proposed system has been carried out by considering various recovery parameters Acc, Rc, Sp, Pr, F1, F2, G1, G2, MCC, ROC curves, and have given satisfactory results here compared to existing methods. This paper concludes that the proposed method has been evaluated on 3000 face images of 15 individuals which are captured using a 5-megapixel low-resolution camera under different conditions. This may lead to the use of different dimension reduction techniques in the future to evaluate the face images of even more 
individuals. Also in the future, the same proposed method needs to be evaluated with additional parameters for the face images of even more individuals for real-world applications.

\section{ACKNOWLEDGEMENTS}

This work has been supported by the Research Center of the Department of Electronics and Communication Engineering, ACS College of Engineering [Research Centre-Visveswaraya Technological University], Bengaluru, Karnataka, India.

\section{REFERENCES}

[1] M. Saini and A. K. Kapoor, "Biometrics in Forensic Identification: Applications and Challenges," Journal of Forensic Medicine, vol. 1, no. 2, pp. 1-6, May.2016, doi: 10.4172/2472-1026.1000108.

[2] K. Gunasekaran, J. Raja and R. Pitchai, "Deep multimodal biometric recognition using contourlet derivative weighted rank fusion with a human face, fingerprint and iris images," Automatika Journal for Control, Measurement, Electronics, Computing and Communications, vol. 60, no. 3, pp. 253-265, Jun. 2019, doi: 10.1080/00051144.2019.1565681.

[3] S. Singh and S. Prasad, "Techniques and Challenges of Face Recognition: A Critical Review," Procedia Computer Science, vol. 143, pp. 536-543, Jan. 2018, doi:10.1016/j.procs.2018.10.427

[4] M. Sharif, F. Naz, M. Yasmin, M. A. Shahid and A. Rehman, "Face Recognition: A Survey," Journal of Engineering Science and Technology Review, vol. 10, no. 2, pp. 166-177, Mar. 2017, doi: 10.25103/jestr.102.20.

[5] M. Chihaoui, A. Elkefi, W. Bellil and C. B. Amar, "A Survey of 2D Face Recognition Techniques," MDPI Journal: Computers, vol. 5, no. 4, pp. 1-8, Sep. 2016, DOI: 10.3390/computers5040021.

[6] Y. Kortli, M. Jridi, A. A. Falou and M. Atri, "Face Recognition Systems: A Survey," MDPI journal: Sensors, vol. 20, no. 2, pp. 1-36, Jan. 2020, doi: 10.3390/s20020342.

[7] A. Lumini, L. Nanni and S. Brahnam, "Ensemble of texture descriptors and classifiers for face recognition," Internation Journal of Applied Computing and Informatics, vol. 13, no. 13, pp.79-91, Jan. 2017, doi: https://doi.org/10.1016/j.aci.2016.04.001

[8] J. Efendi, M. I. Zul and W. Yunanto, "Real-Time Face Recognition using Eigenface and Viola-Jones Face Detector," International journal on informatics visualization, vol. 7, no. 1, pp. 16-22, Mar. 2017, doi: 10.30630/joiv.1.1.15.

[9] M. Castrillon, O. Deniz, D. Hernandez and J. Lorenzo, "A comparison of the face and facial feature detectors based on the Viola-Jones general object detection framework," Journals of Machine Vision and Applications, Springer, vol. 22, no. 3, pp. 481-494, May. 2011, doi: 10.1007/s00138-010-0250-7.

[10] P. Irgens, C. Bader, T. Lé, D. Saxena and C. Ababei, "An efficient and cost-effective FPGA-based implementation of the Viola-Jones face detection algorithm," HardwareX, Elsevier Journals, vol. 1, pp. 68-75, Mar. 2017, doi: 10.1016/j.ohx.2017.03.002.

[11] A. A. Ali, T. A. El-Hafeez and Y. K. Mohany, "An Accurate System for Face Detection and Recognition," Journal of Advances in Mathematics and Computer Science, vol. 33, no. 3, pp. 1-19, Jul. 2019, doi: 10.9734/jamcs/2019/v33i330178.

[12] X. Gao, F. Sattar and R. Venkateswarlu, "Multiscale corner detection of gray-level images based on log-Gabor wavelet transform," IEEE Transactions on Circuits and Systems for Video Technology [ICASSP], vol. 17, no. 7, pp. 868-875, Aug. 2017, doi: 10.1109/TCSVT.2007.897473.

[13] M. Elsayed, R. M. Farouk and M. Aly, "Medical Image Denoising based on Log-Gabor Wavelet Dictionary and KSVD Algorithm," International Journal of Computer Applications, vol. 141, no. 1, pp. 27-32, May. 2017, doi: 10.5120/ijca2016909209.

[14] M. A. R. Ratul, S. A. Raja and J. Uddin, "A Novel Parallel Texture Feature Extraction Method using Log-Gabor Filter and Singular Value Decomposition (SVD)," ICCMS 17: Proceedings of the 8th International Conference on Computer Modeling and Simulation, pp.187-191, Jan. 2017, doi: 10.1145/3036331.3036352.

[15] S. Allagwail, O. S. Gedik and J. Rahebi, "Face Recognition with Symmetrical Face Training Samples Based on Local Binary Patterns and the Gabor Filter," MDPI Journal: Symmetry, vol. 11, no. 2, pp. 1-22, Jan. 2019, doi: 10.3390/sym 11020157 .

[16] T. Ayyavoo and J. J. Suseela, "Illumination pre-processing method for face recognition using 2D DWT and CLAHE," IET Biometrics, vol. 7, no. 4, pp. 380-390, Oct. 2017, doi: 10.1049/iet-bmt.2016.0092.

[17] B. Sujatha et al., "Translation based Face Recognition using Fusion of LL and SV Coefficients," Twelfth International Multi-Conference on Information Processing-2016 (IMCIP-2016), Procedia Computer Science 89, pp. 877-886, Dec. 2016, doi: 10.1016/j.procs.2016.06.077.

[18] K. S. Roopashri and M. Kulkarni, "An Efficient Face Recognition using DWT and FFT Algorithm," IJSTE International Journal of Science Technology \& Engineering, vol. 2, no. 12, pp. 255-258, Jul. 2016.

[19] A. Benzaoui, A. Boukrouche, H. Doghmane and H. Bourouba, "Face recognition using 1DLBP, DWT and SVM," 3rd IEEE International Conference on Control, Engineering \& Information Technology (CEIT), pp. 1-6, May. 2015, doi: 10.1109/CEIT.2015.7233002.

[20] M. A. Al-Abaji and M. M. Salih, "The Using Of PCA, Wavelet and GLCM In Face Recognition System," Journal of the University of Babylon for Pure and Applied Sciences, vol. 26, no. 10, pp. 131-139, Dec. 2018, doi: 10.29196/jubpas.v26i10.1848. 
[21] S. Setumin, Shahrel and A. Suandi, "Difference of Gaussian Oriented Gradient Histogram for Face Sketch to Photo Matching," IEEE Access, vol. 6, pp. 39344-39352, Jul. 2018, doi: 10.1109/ACCESS.2018.2855208.

[22] S. Anila and N. Devarajan, "Preprocessing Technique for Face Recognition Applications under Varying Illumination Conditions," Global Journal of Computer Science and Technology Graphics \& Vision, vol. 12, no. 11, pp.12-18, Jan. 2012.

[23] M. W. Davidson and M. Abramowitz, "Molecular Expressions Microscopy Primer: Digital Image ProcessingDifference of Gaussians Edge Enhancement Algorithm," Olympus America Inc. and Florida State University: Miami, FL, USA, 2006.

[24] R. González and R. Woods, "Digital image processing," Pearson 3rd Edition, 2008.

[25] M. Sun, "A Multiclass support vector machine: theory and model," International Journal of Information Technology \& Decision Making, vol. 12, no. 6, pp. 1175-1199, Nov. 2013, doi: 10.1142/S0219622013500338.

[26] P. Sun, D. Wang, V. C. Mok and L. Shi, "Comparison of Feature Selection Methods and Machine Learning Classifiers for Radiomics Analysis in Glioma Grading," IEEE Access, vol. 7, pp. 102010-102020, Jul. 2019, doi: 10.1109/ACCESS.2019.2928975.

[27] F. M. da Silva, "Notes on Support Vector Machines," Neural Network Group, I N E S C, pp. 1-60, 1998.

[28] D. Mahmoodi, A. Soleimani, H. Khosravi, and M. Taghizadeh," FPGA Simulation of Linear and Nonlinear Support Vector Machine," Journal of Software Engineering and Applications, vol. 4, no. 5, pp. 320-328, Jan. 2011, doi: 10.4236/jsea.2011.45036.

[29] M. J. J. Ghrabat, G. Ma, I. Y. Maolood, S. S. Alresheedi and Z. A. Abduljabbar, "An effective image retrieval based on optimized genetic algorithm utilized a novel SVM-based convolutional neural network classifier," Human-centric Computing and Information Sciences, Article no. 31, pp. 1-29, Dec. 2019, doi: 10.1186/s13673019-0191-8.

[30] P. Viola and M. J. Jones, "Rapid object detection using a boosted cascade of simple features," Proceedings of the 2001 IEEE Computer Society Conference on Computer Vision and Pattern Recognition (CVPR), vol. 1, pp. 511518, Dec. 2001, doi: 10.1109/CVPR.2001.990517.

\section{BIOGRAPHIES OF AUTHORS}
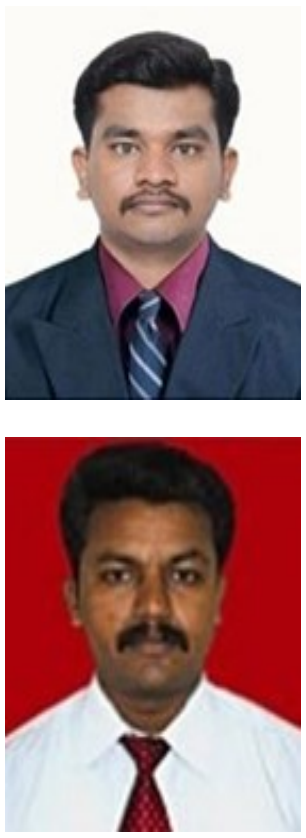

Vijaya Kumar H. R. received his undergraduate degree in the Department of Electronics and Communication from Visvesvaraya Technological University, Karnataka, India, in 2009 and a Postgraduate degree in Digital electronics from Sri Siddhartha University, Tumakuru, Karnataka, India in 2011. He is currently working as an Assistant Professor in the Department of Electronics and Communication Engineering, Akshaya Institute of Technology, Tumkuru, Karnataka and currently pursuing his Ph.D. in the image processing field from 2016 in the Research Centre, Department of Electronics and Communication Engineering, ACS College of Engineering, Bengaluru, under Visvesvaraya Technological University (VTU- RC), Karnataka, India.

Dr. M. Mathivanan received his Undergraduate in the Department of Electronics and Communication from University of Madras, Tamilnadu, India, in 2001, and Postgraduate degree in Applied Electronics from Anna University, Tamilnadu, India in 2007 respectively, and the Ph.D. degree from Anna University, Tamilnadu, India in 2014. He is the author/coauthor of over 20 referred research papers. He is currently working as a Research guide in the Department of Electronics and Communication Engineering, ACS College of Engineering, Bengaluru, Karnataka, India. His research areas cover Signal Processing, Speech and Image Processing, Embedded systems. 